\title{
Estenose degenerativa do canal lombar
}

\section{Degenerative Lumbar Spinal Stenosis}

\author{
Sergio Hennemann ${ }^{1}$ Marcelo Rodrigues de Abreu ${ }^{2}$ (D) \\ ${ }^{1}$ Serviço de Ortopedia, Grupo da coluna, Hospital Mãe de Deus, Porto \\ Alegre, RS, Brasil \\ 2 Radiologia Musculoesquelética, Hospital Mãe de Deus, Porto Alegre, \\ Endereço para correspondência Sergio Hennemann, Rua Costa 30/806, \\ RS, Brasil \\ Porto Alegre, RS, 90110270, Brasil (e-mail: sergiohe@terra.com.br). \\ Rev Bras Ortop 2021;56(1):9-17.
}

\section{Resumo \\ Palavras-chave \\ - artrodese \\ - claudicação intermitente \\ - descompressão \\ - estenose \\ - dor lombar}

Abstract
A estenose degenerativa do canal vertebral lombar é a causa mais frequente de dor lombar e/ou ciática no paciente idoso; sua epidemiologia, fisiopatogenia, manifestações e testes clínicos são revistos em ampla investigação bibliográfica atual. A importância da relação entre a clínica e o estudo por imagens, principalmente a ressonância magnética (RM), é ressaltada. Antes da indicação do tratamento, é necessário identificar a localização precisa da dor, bem como o diagnóstico diferencial entre a claudicação neurogênica e a vascular. $O$ tratamento conservador associando medicações com as diversas técnicas fisioterápicas resolve o problema na maioria dos casos, já o teste terapêutico com os bloqueios, seja epidural, foraminal ou facetário, é realizado quando as dores não cedem com o tratamento conservador e antes da indicação da cirurgia. Os bloqueios costumam dar melhores resultados no alívio dos sintomas de ciatalgia e menos no quadro de claudicação neurogênica. A descompressão da cauda equina e/ou radicular associada ou não à artrodese é o padrão ouro quando a intervenção cirúrgica é necessária. A artrodese após a descompressão é necessária nos casos com instabilidade segmentar, como na espondilolistese degenerativa. Quando a estenose de canal acontece em múltiplos níveis e vem acompanhada de desvio de eixo, seja coronal e/ou sagital, deve ser realizada, além das descompressões e artrodese, a correção dos desvios de eixo, principalmente o eixo sagital, quando a correção da lordose lombar se impõe com técnicas que corrigem a lordose retificada para valores próximos à incidência pélvica.

Degenerative lumbar spinal stenosis is the most frequent cause of low back pain and/or sciatica in the elderly patient. Epidemiology, pathophysiology, clinical manifestations and testing are reviewed in a wide current bibliographic investigation. The importance of the relationship between clinical presentation and imaging study, especially magnetic resonance imaging (MRI), is emphasized. Prior to treatment indication, it recebido

12 de Novembro de 2019

aceito

02 de Março de 2020

Publicado online

Julho 23, 2020
DOI https://doi.org/

10.1055/s-0040-1712490. ISSN 0102-3616. (c) 2020. Sociedade Brasileira de Ortopedia e Traumatologia. All rights reserved.

This is an open access article published by Thieme under the terms of the Creative Commons Attribution-NonDerivative-NonCommercial-License, permitting copying and reproduction so long as the original work is given appropriate credit. Contents may not be used for commercial purposes, or adapted, remixed, transformed or built upon. (https://creativecommons.org/ licenses/by-nc-nd/4.0/)

Thieme Revinter Publicações Ltda., Rua do Matoso 170, Rio de Janeiro, RJ, CEP 20270-135, Brazil 


\section{Keywords}

- arthrodesis

- intermittent claudication

- decompression

- stenosis

- low back pain is necessary to identify the precise location of pain, as well as the differential diagnosis between neurological and vascular lameness. Conservative treatment combining medications with various physical therapy techniques solves the problem in most cases, while therapeutic testing with injections, whether epidural, foraminal or facetary, is performed when pain does not subside with conservative treatment and before surgery is indicated. Injections usually perform better results in relieving sciatica symptoms and less in neurological lameness. Equine tail and/or root decompression associated or not with fusion is the gold standard when surgical intervention is required. Fusion after decompression is necessary in cases with segmental instability, such as degenerative spondylolisthesis. When canal stenosis occurs at multiple levels and is accompanied by axis deviation, whether coronal and/or sagittal, correction of axis deviations should be performed in addition to decompression and fusion, especially of the sagittal axis, in which a lumbar lordosis correction is required with techniques that correct the rectified lordosis to values close to the pelvic incidence.

\section{Introdução}

A estenose de canal lombar é definida como todo e qualquer estreitamento do canal vertebral, dos forames ou do recesso lateral, levando a quadro clínico de dor lombar, a qual pode irradiar para nádegas e membros inferiores e apresenta causas desencadeantes e de alívio bem definidas, decorrentes da compressão das estruturas neurovasculares no interior do canal lombar.

\section{Causas de estenose do canal vertebral}

A. Alterações congênitas, como pedículos curtos ou orientação anômala das facetas articulares.

B. Causas adquiridas:

1. Como consequência de traumatismos: fratura de vértebra com fragmento ósseo projetado para dentro do canal, luxação vertebral;

2. Tumores ósseos ou metástases de tumores de partes moles que podem invadir o canal;

3. Hematomas de diversas origens;

4. Abscesso decorrente de processo infeccioso com origem no corpo vertebral ou nos discos intervertebrais;

5. Várias doenças ósseo-metabólicas ou endócrinas como acromegalia, osteodistrofia renal e hipoparatireoidismo;

6. Outras doenças ósseas deformantes como doença de Paget, acondroplasia, artrite reumatoide, espondilite anquilosante e hiperostose óssea idiopática difusa;

7. Causas iatrogênicas: pós-cirurgia de descompressão ou de ressecção óssea

8. Doença degenerativa por discopatia degenerativa e artrose facetária: a mais comum de todas.

C. Causas mistas: estenose congênita associada a estenose adquirida: por exemplo, estenose de canal por pedículos curtos associada a discoartrose.

Na presente revisão, nos ateremos à estenose de canal lombar de origem degenerativa.

\section{Epidemiologia}

Apesar de o processo degenerativo acometer a coluna vertebral de forma quase universal após os 50 a 60 anos de vida, apenas $\sim 6 \%$ das pessoas adultas sofrem de estenose de canal lombar sintomática. ${ }^{1,2}$

Quando a estenose de canal degenerativa se associa às causas congênitas (como pedículo curto ou orientação sagital das facetas articulares), as manifestações clínicas podem ocorrer já na faixa dos 30 aos 40 anos de vida. ${ }^{3}$

A estenose de canal lombar representa a causa mais comum das patologias da coluna lombar em pacientes $>65$ anos de idade que necessitam de tratamento cirúrgico, e estima-se que $\sim 0,1 \%$ da população vá necessitar de algum procedimento para tratar de problemas degenerativos da coluna lombar. ${ }^{4}$

\section{Anatomia do canal}

Os principais limites do canal vertebral são: anteriormente, o disco intervertebral e o corpo vertebral; lateralmente, os dois pedículos e as articulações interapofisárias com as suas cápsulas; posteriormente, as lâminas e o ligamento amarelo.

Na estenose central, ocorre o estreitamento do canal, anteriormente, pela protrusão do disco intervertebral e dos osteófitos da região posterior dos corpos vertebrais e, posteriormente, pela indentação do ligamento amarelo, que se apresenta espessado. 0 espaço do canal vertebral diminui com a extensão da coluna lombar e aumenta com a sua flexão, o que caracteriza o componente dinâmico dos sintomas da estenose de canal. ${ }^{5}$

$\mathrm{Na}$ estenose do recesso lateral, vemos a hipertrofia das cápsulas articulares interapofisárias e, nos estados mais avançados, a projeção dos osteófitos para o interior do canal a partir das facetas superiores da vértebra inferior são a principal causa, resultando na compressão do saco dural e da raiz transeunte.

Na estenose foraminal, a protrusão do disco intervertebral se associa ao osteófito que se forma a partir da apófise articular superior da vértebra inferior, vindo a comprimir a raiz nervosa emergente. Isso ocorre com maior frequência na coluna lombar baixa, onde o diâmetro do forâmen diminui anatomicamente ao mesmo tempo em que a raiz nervosa aumenta de 

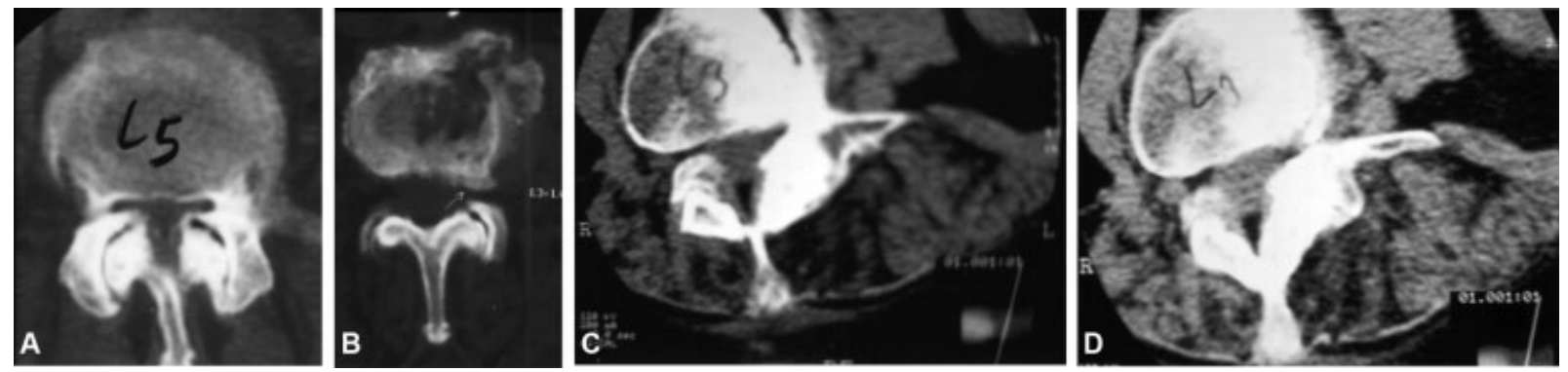

Fig. 1 Cortes axiais de tomografia computadorizada mostrando: Estenose central, de (A) recesso lateral e (B) foraminal; (C e D) Escoliose degenerativa com rotação vertebral e laterolistese com estenose foraminal.

diâmetro, tornando a coluna mais facilmente sujeita a compressões, mesmo por osteófitos menores (- Figura $\mathbf{1}$ ).

\section{Clínica e história natural}

A maior parte dos casos de estenose torna-se sintomática a partir da sexta década de vida, e os sintomas, com início geralmente insidioso, costumam estar relacionados com a degeneração que ocorre no nível de L3-L4 e L4-L5. Na maioria das vezes, em uma fase inicial, há queixas de dor lombar de repetição que, com o tempo, se torna permanente. Esse sintoma geralmente está relacionado com a degeneração discal, em suas diversas fases, e com o início da artrose facetária, caracterizada por sinovite.

Em sua evolução, a dor lombar pode se irradiar para os flancos e a região glútea e, eventualmente, para o trajeto da raiz nervosa, quando fica caracterizada a provável associação com hérnia de disco ou mesmo com a estenose foraminal ou do recesso lateral.

Quando a estenose é central, o sintoma clássico é a claudicação neurogênica, que consiste em dor nos membros inferiores, parestesias e diminuição de força, com progressão lenta e insidiosa. Esses sintomas estão associados com o caminhar ou o ficar de pé e aliviam quando o paciente está sentado, inclinando-se para frente ou deitado. ${ }^{6}$

Para melhor compreensão dos sintomas do paciente, temos que nos reportar à cascata degenerativa descrita por Kirkaldy-Willis et al, ${ }^{7}$ que descrevem a evolução do processo degenerativo tanto no nível dos discos intervertebrais como no das facetas articulares e dos corpos vertebrais.

Uma possível causa da estenose lombar degenerativa é a orientação sagital das facetas articulares, a qual também constitui causa de estenose congênita. ${ }^{8}$

Numa fase final do processo degenerativo, advém a anquilose do(s) segmentos(s) comprometidos(s) e, clinicamente, pode ocorrer agravamento dos sintomas, com dor mesmo em repouso, e até alteração neurológica mais severa, como síndrome da cauda equina ou bexiga neurogênica.

\section{Exame físico}

Os achados do exame físico, na estenose de canal central, costumam ser pobres e pouco característicos.

Os sintomas da claudicação podem se manifestar quando o examinador solicita que o paciente caminhe ou hiperes- tenda a coluna. No estágio avançado, muitas vezes se verifica a retificação da lordose lombar e a perda do equilíbrio sagital, em que o paciente se encontra curvado para frente.

Eventualmente, o paciente pode apresentar sintomas e sinais radiculares em quadros de estenose foraminal, ou hérnia de disco associada aos sinais radiológicos de estenose do forâmen ou do recesso lateral. Na maioria das vezes, não encontramos sinal de irritação radicular ou sinal de Laségue positivo.

As eventuais diminuições de força de determinados grupos musculares e as correspondentes alterações da sensibilidade e dos reflexos, com distribuição metamérica, podem estar presentes mais raramente, dependendo da localização e do grau de estenose do canal lombar.

Conforme as conclusões de um grupo de 279 especialistas de 29 países, no sentido de obter um consenso internacional, existiriam 7 sinais e sintomas clínicos que fazem ter $80 \%$ de certeza da estenose de canal lombar na história e no exame físico, a saber: dor na região glútea ou nos membros inferiores ao caminhar; flexão anterior para aliviar os sintomas; sentir alívio ao usar carrinho de supermercado ou andar de bicicleta; distúrbios sensitivos ou motores ao caminhar; pulsos periféricos normais ou simétricos; fraqueza nas extremidades inferiores; e dor lombar. ${ }^{9}$

Uma eventual concomitância da estenose de canal lombar com estenose de canal cervical ou dorsal pode ocorrer, e nesse caso predominam os sinais de radiculopatia cervical ou de mielopatia, que se caracteriza pela presença de espasticidade, hiperreflexia, clonus e perda de equilíbrio.

\section{Diagnóstico diferencial entre claudicação neurogênica e vascular}

O diagnóstico diferencial entre claudicação neurogênica e vascular deve ser definido na ocasião do exame físico do paciente. $O$ tratamento só deve ser iniciado depois da realização desse diagnóstico diferencial.

A claudicação de etiologia vascular (isquêmica), na maioria dos casos, se apresenta com dor na panturrilha, que pode se estender proximalmente. Os pulsos das artérias das extremidades inferiores geralmente estão diminuídos, devendo ser pesquisados por palpação ou ausculta os pulsos das artérias aorta, femoral, poplítea, tibial posterior e pediosa.

Geralmente, o paciente refere que os sintomas são agravados e aliviados por atividades em qualquer posição. 0 
alívio não tem relação com a posição de flexão ou extensão. Ao pedalar na bicicleta, os sintomas da estenose de origem vascular se agravam, o que não acontece em quadros de estenose neurogênica, devido à flexão do tronco.

Em caso de suspeita de alterações de origem vascular, devem ser pedidos exames específicos de investigação da circulação vascular periférica arterial e venosa.

É importante também, nos pacientes idosos, excluir a possibilidade de neuropatias periféricas, principalmente nos diabéticos. As características principais dessa situação são hipoestesia em "bota", redução da sensibilidade vibratória, presença de "queimação" noturna e falta de correlação com a atividade física. Nessa circunstância, para estabelecer o diagnóstico diferencial, é importante solicitar o exame de eletroneuromiografia.

Delamarter et $\mathrm{al}^{10}$ e Rydevik et $\mathrm{al}^{11}$ descreveram as alterações eletrofisiológicas das raízes nervosas decorrentes da congestão vascular provocada por uma compressão extrínseca, como ocorre na estenose do canal vertebral. Com isso, os autores quiseram demonstrar que os sintomas e sinais na estenose ocorreriam mais pelo distúrbio de vascularização das estruturas nervosas associado às alterações inflamatórias do que pela compressão em si. Assim, pode-se concluir que os sintomas e sinais decorrentes do quadro de estenose degenerativa do canal advêm de uma soma de alterações mecânicas, vasculares e neurológicas.

\section{Avaliações por imagem da estenose do canal}

A estenose do canal lombar é uma síndrome clínico-radiológica, sendo necessário o fornecimento das seguintes informações pelos radiologistas em caso de suspeita dessa síndrome: primeiro, se o paciente preenche ou não os critérios de estenose; segundo, se houver estenose, devem ser fornecidas informações detalhadas sobre a localização da estenose e o(s) fator(es) causador(es) dela. Essas informações são necessárias para recomendar opções de tratamento adequadas.

A North American Spine Society afirma, em suas diretrizes, que a imagem é o principal exame não invasivo para o diagnóstico da estenose espinhal lombar, mas não fornece critérios radiológicos para a estenose. Especialistas em radi- ologia musculoesquelética usam principalmente critérios qualitativos para o diagnóstico de estenose espinhal lombar. Genevay et al $^{12}$ relataram que vários critérios são usados para descrever a estenose espinhal lombar, mas que nem sempre são claramente definidos e podem impedir um diagnóstico confiável.

Critérios qualitativos utilizados para o diagnóstico de estenose espinhal lombar incluem a presença de:

- Protrusão de disco

- Apagamento da gordura perineural

- Degeneração da articulação facetária com hipertrofia

- Ausência de fluido ao redor da cauda equina

- Hipertrofia do ligamento amarelo

- Redundância das raízes da cauda equina e forma serpentiginosa

- Lipomatose epidural ${ }^{13}$ (-Figura 2A)

\section{Exame radiológico simples}

A estenose do canal vertebral já pode ser fortemente suspeitada em radiografias simples, quando são realizadas em pacientes com dor nas costas. O diâmetro anteroposterior (AP) do canal vertebral aumenta caudalmente e deve ser considerado anormal se for $<12 \mathrm{~mm}$ na coluna lombar ${ }^{14}$ $\mathrm{e}<10 \mathrm{~mm}$ na coluna cervical. ${ }^{15,16}$

\section{Ressonância magnética e tomografia}

A ressonância magnética (RM) é sugerida como o teste não invasivo mais apropriado para confirmar a presença de estreitamento anatômico do canal vertebral ou de impacto radicular em pacientes com suspeita clínica de estenose espinhal lombar. Com base no diâmetro anteroposterior do canal medular ou na área da seção transversal do saco dural, pode-se diagnosticar a estenose do canal lombar.

A área transversal do saco dural é considerada normal se for $>100 \mathrm{~mm}^{2}$ no ponto mais estreito, estenótica se medir entre 76 e $100 \mathrm{~mm}^{2}$ e gravemente estenótica se for $<76 \mathrm{~mm}^{2}$. A RM e a tomografia computadorizada (TC) têm a vantagem de possibilitar a visualização direta do canal central e lateral. A RM tem o benefício adicional de possibilitar a visualização de tecido mole. ${ }^{17}$
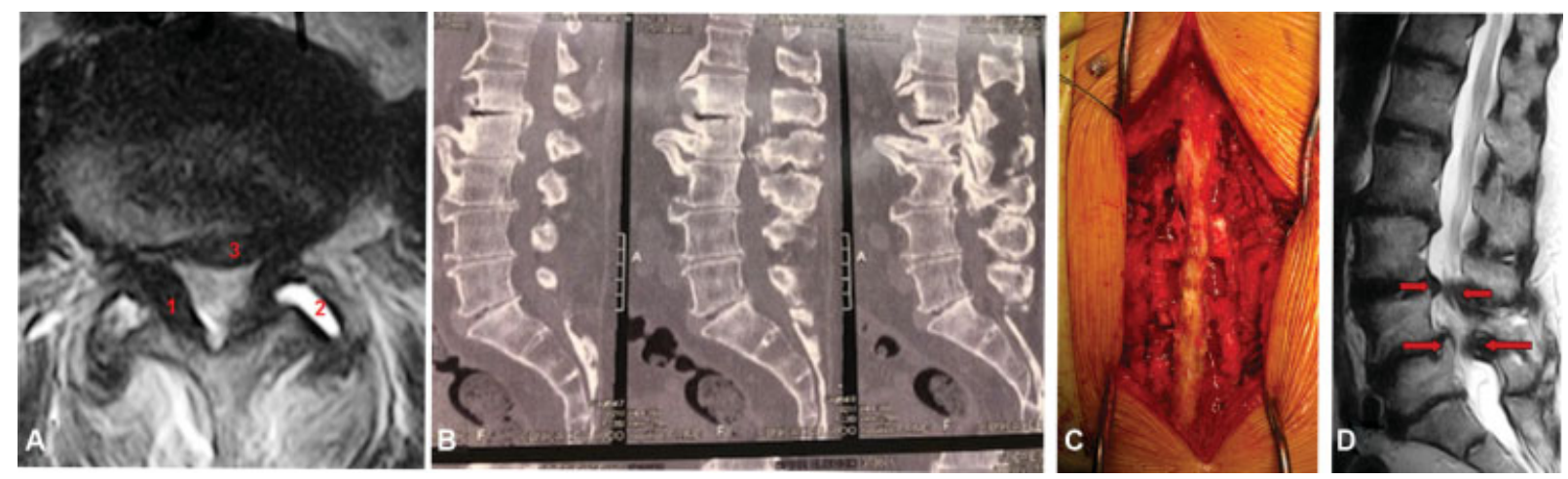

Fig. 2 (A) Corte axial de ressonância magnética mostrando estenose de canal com espessamento do ligamento amarelo (1), artrose facetaria com sinovite (2) e protusão discal (3). (B) Corte sagital de tomografia computadorizada mostrando estenose de canal em L1-L2-L3-L4. (C) Foto da cirurgia: descompressão nos três níveis com técnica convencional de laminectomia + ressecção do recesso lateral e foraminotomia bilateral. (D) Corte sagital de ressonância magnética mostrando estenose degenerativa do canal L3-L4 e L4-L5 com espondilolistese degenerativa em L4-L5. 


\section{Avaliação do canal vertebral, critérios diagnósticos}

- Diâmetro AP do canal ósseo $<10 \mathrm{~mm}$ na coluna cervical ou $<12 \mathrm{~mm}$ na coluna lombar;

- A área da seção transversal do saco dural no ponto mais estreito é considerada estenótica se medir entre 76 e $100 \mathrm{~mm}^{2}$ e gravemente estenótica se for $<76 \mathrm{~mm}^{2}$.

Avaliação do neuroforâmen e do recesso lateral, critérios diagnósticos

- Diâmetro AP do forâmen $<3 \mathrm{~mm}$ nas imagens sagitais é considerado diagnóstico para estenose;

- Altura do recesso lateral $<3 \mathrm{~mm}$ ou ângulo lateral do recesso $<30^{\circ}$ são também evidências de estenose espinhal.

\section{Estenose funcional do canal}

A estenose do canal funcional é mais importante do que a estenose óssea do canal vertebral na apresentação clínica. ${ }^{14} \mathrm{~A}$ estenose do canal funcional é produzida por vários componentes de tecidos moles, tais como protrusão discal; ligamentos hipertróficos; cistos sinoviais; e instabilidades, que determinam os sintomas e manifestações neurológicas, muitas vezes não demonstrados no estudo por imagem. Estudos em posição ortostática (ressonância ortostática) e estudos funcionais (radiografias dinâmicas) podem contribuir para melhor firmar o diagnóstico de estenose por imagem.

Exames mais recentes de neurografia (ressonância especializada em nervos) podem fornecer informações específicas quantitativas sobre alterações fisiológicas das raízes e de seus gânglios por meio da difusão da água dos axônios, e podem contribuir para melhor entendimento dos sintomas e da correlação clínica.

\section{Estenose de canal lombar: tratamento conservador}

Em 1993, Onel et al ${ }^{17}$ publicaram sua experiência de tratamento conservador, em um estudo prospectivo que incluía 145 pacientes com estenose de canal lombar.

O tratamento conservador consistiu em fisioterapia com termoanalgesia + exercícios + uso de calcitonina. Os pacientes apresentaram sensível melhora, com resultados estatisticamente significativos, com exceção daqueles relacionados a alterações no reflexo profundo. Os autores concluíram que o tratamento conservador pode ser o tratamento de escolha em pacientes mais idosos e naqueles sem condições clínicas para a cirurgia de descompressão.

Desde que o quadro de claudicação neurogênica não seja grave e não haja sintomatologia de déficit motor, o tratamento inicial deve visar o alívio da dor com repouso e mudança das atividades do dia a dia. Recomenda-se, inicialmente, o uso de analgésicos e anti-inflamatórios não esteroides, que podem ser associados a relaxantes musculares. Para obter alívio das dores, nessa fase, pode-se associar fisioterapia com termoanalgesia, estimulação nervosa transcutânea (TENS, na sigla em inglês) e exercícios leves de mobilização com alongamentos e reforço muscular progressivo de correção postural. A acupuntura, a quiropraxia e os exercícios indicados pelo método McKenzie também podem ser utilizados para obter o alívio das dores. Nenhum desses métodos mostra comprovada superioridade de um sobre outro, e nenhum proporciona melhora importante do quadro de claudicação neurogênica. ${ }^{18,19}$

Se houver história de dor crônica, parestesias, disestesias, ou dor do tipo neuropático nos membros inferiores, esse tratamento pode ser associado ao uso de antidepressivos tricíclicos e/ou anticonvulsivantes como a gabapentina ou a pregabalina.A pregabalina é a droga de escolha na dor neuropática - $40 \%$ dos pacientes sentem alívio. ${ }^{20}$

Os analgésicos, anti-inflamatórios e anticonvulsivantes também não demonstram grande eficácia na melhora do sintoma de claudicação neurogênica. Os corticoesteroides podem ter seu uso indicado em caso de agudização dos sintomas de irritação radicular, sempre de forma breve para diminuir os riscos de efeitos colaterais, assim como o uso de analgésicos narcóticos.

O bloqueio epidural com injeções de corticoide no espaço epidural traz alívio dos sintomas da estenose de canal, mais para os sintomas de radiculopatias e menos para a claudicação neurogênica, apesar de não haver estudos que demonstrem a eficácia desse tratamento por tempo prolongado. Riew et al relatam que $71 \%$ dos pacientes que inicialmente concordavam com a cirurgia desistiram dela após serem submetidos a injeção seletiva de raízes nervosas com betametasona e bupivacaína. ${ }^{21}$

Esses bloqueios podem ser realizados por via interlaminar ou por acesso caudal e, quando predominam as dores radiculares, se indica injeção seletiva transforaminal na raiz nervosa com corticoide e bupivacaína, realizada sob orientação fluoroscópica.

\section{Tratamento conservador ou cirúrgico?}

Um estudo realizado por Johnsson et $\mathrm{al}^{22}$ observou o resultado do tratamento conservador em um grupo de 49 pacientes e revelou que apenas $18 \%$ dos pacientes necessitaram de tratamento cirúrgico.

Zaina et $\mathrm{al}^{23}$ avaliaram a efetividade das diferentes técnicas cirúrgicas comparadas com os diferentes tipos de tratamento conservador da estenose de canal lombar e concluíram ser impossível afirmar qual tratamento (cirúrgico ou conservador) é o melhor, devido à grande variedade de condutas nos tratamentos realizados.

O paciente tende a optar mais pelo tratamento cirúrgico quando a apresentação clínica está agravada por sintomas de comprometimento radicular, como na estenose do recesso lateral. ${ }^{24}$

Os resultados da descompressão cirúrgica com ou sem artrodese associada se mostram superiores nos primeiros anos de pós-operatório e tendem a convergir após 8 anos de tratamento cirúrgico. ${ }^{25,26}$

Com base nos diversos estudos, pode-se concluir que o tratamento conservador é o tratamento de escolha em uma fase inicial de tratamento tanto no quadro de sintomas radiculares como no de claudicação neurogênica, desde que não haja comprometimento neurológico com déficit motor com piora progressiva na primeira situação, ou claudicação no teste 
de pequeno trajeto de caminhada na segunda situação. Nessas circunstâncias, é recomendado o tratamento cirúrgico. ${ }^{27}$

\section{Tratamento cirúrgico}

O tratamento cirúrgico da estenose de canal lombar tem indicação nos casos de falha do tratamento conservador. Também está indicado nos casos em que a sintomatologia já de início é muito aguda, com comprometimento radicular associado a sintomas de alterações sensitivas e motoras de dermátomo com agravamento progressivo ou a sintomas de claudicação neurogênica severa. Nessas circunstâncias, os sintomas devem ser relacionados com os achados do estudo por imagem, que irá guiar o tipo de cirurgia a ser realizada quanto ao segmento e à área que vai necessitar da descompressão.

É importante, também, avaliar a necessidade de descompressão e artrodese na situação de instabilidade clínica e/ou radiológica, principalmente quando, no estudo por imagem, há o diagnóstico de espondilolistese degenerativa. 0 estudo da necessidade de descompressão associada a artrodese e à correção de deformidades do eixo tanto coronal como sagital se impõe no momento do planejamento estratégico para a intervenção cirúrgica. $O$ objetivo do tratamento cirúrgico é a melhora da função, o alívio da dor e a redução ou prevenção do déficit neurológico. Para isso, se impõe a descompressão das estruturas neurais, cuja extensão será determinada pelos sinais e sintomas e pelo estudo por imagem de cada caso. Mesmo quando a dor lombar é importante em comparação aos sintomas radiculares, se obtém alívio das dores na maioria dos casos submetidos a descompressão. ${ }^{28}$

A descompressão do canal vertebral pode ser realizada por diversas técnicas. $O$ padrão ouro continua sendo a técnica aberta por laminectomia ou laminotomia, na qual, pela ressecção ou abertura das lâminas, seguida da ressecção do ligamento amarelo, que geralmente se encontra espessado, se expõem as estruturas nervosas sob compressão. A laminotomia pode ser unilateral, bilateral ou por divisão do processo espinhoso. Esta última diminui a agressão sobre a musculatura paravertebral, reduzindo assim as complicações pós-operatórias relacionadas a hematomas, seromas e infecções e à atrofia da musculatura extensora do tronco. ${ }^{29,30}$ o saco dural é descomprimido após a sua exposição e afastado para permitir a ressecção do recesso lateral e a abertura do forâmen para descompressão da raiz nervosa transeunte e /ou emergente (-Figuras 2B e 2C). Essa descompressão também pode ser realizada por técnicas minimamente invasivas. ${ }^{31}$

Em caso de necessidade de descompressão foraminal bilateral, isso pode ser realizado de diversas maneiras: por abertura bilateral; abertura unilateral para alcançar ambos os recessos laterais e ambos os foramens; ou pela abordagem unilateral, alcançando-se o lado contralateral pela técnica "over the top" endoscópica (-Figura 2). ${ }^{32}$

Existe, atualmente, muita discussão quanto aos resultados das técnicas abertas em comparação com as minimamente invasivas. As evidências das revisões sistemáticas e de metanálises sugerem que a técnica de laminectomia unilateral para descompressão bilateral minimamente invasiva é associada a menor perda sanguínea e a menor tempo de hospi- talização, com perfil de complicações similar ao da técnica aberta e com resultado semelhante a longo prazo. ${ }^{33}$

A estenose, quando puramente foraminal, pode ser resolvida cirurgicamente por técnica aberta por laminectomia ou por técnica endoscópica percutânea com ressecção de osteófitos ou associada a discectomia. ${ }^{34}$

\section{Cirurgia de descompressão e artrodese}

A necessidade de artrodese após descompressão por estenose de canal lombar depende basicamente da presença de instabilidade segmentar clínica e/ou radiológica.

Devem-se levar em consideração os parâmetros radiológicos quando acompanhados de apresentação clínica indicativa de estenose de canal, lembrando que, algumas vezes, existem essas variações sem sintomatologia importante..$^{35,36}$

É, também, indicada a cirurgia de artrodese associada a descompressão quando esta exige a ressecção de $>50 \%$ das facetas bilateralmente para descomprimir as estruturas neurológicas, o que leva ao risco de instabilidade iatrogênica, ou quando há escoliose degenerativa progressiva, com desvio de eixo coronal e sagital e estenose de canal associada em vários níveis. ${ }^{37}$

\section{Espondilolistese degenerativa}

Em muitos casos sintomáticos de estenose de canal lombar, há a presença da espondilolistese degenerativa no estudo por imagem. $\mathrm{O}$ grau de degeneração facetária e discal que leva à listese é muito variável de caso para caso, e existem diversos graus de escorregamento da vértebra superior sobre a inferior no sentido AP ou mesmo lateral. Essa translação pode levar à compressão de raízes nervosas nos foramens ou no recesso lateral, podendo também diminuir o diâmetro do canal vertebral, levando à estenose central ( - Figura 2D).

Na maioria dos casos em que é necessário tratamento cirúrgico para a espondilolistese degenerativa, basta realizar a descompressão. Quando há necessidade de ressecção facetária mais ampla ou mesmo na evidência de instabilidade clínica ou radiológica presente se impõe a descompressão associada à artrodese. ${ }^{38}$

Uma metanálise da literatura realizada por Martdjetko et al $^{39}$ sobre trabalhos publicados entre 1970 e 1993 relativos à cirurgia por espondilolistese degenerativa demonstrou melhores resultados em pacientes submetidos a descompressão e artrodese, seja por artrodese in situ ou instrumentada, em comparação aos submetidos apenas a descompressão. ${ }^{40}$

Quando se decide pela artrodese associada à descompressão na espondilolistese degenerativa, pode-se realizá-la por via posterolateral aberta ou por técnica minimamente invasiva - fusão intersomática lombar transforaminal (transforaminal lumbar interbody fusion [TLIF, na sigla em inglês]) ou minimally invasive (MIS, na sigla em inglês) TLIF. Isso porque não foram encontradas, nos trabalhos publicados, maiores diferenças nos resultados entre as duas técnicas, a não ser o menor tempo de hospitalização, o menor sangramento e o menor grau de dor na técnica minimamente invasiva (MIS TLIF). ${ }^{41}$ 
A artrodese por técnica mini-open pelo acesso posterolateral transmuscular (técnica de Wiltse), de menor custo e com os mesmos resultados no comparativo com a técnica MIS TLIF, é a de preferência dos autores. ${ }^{42,43}$

\section{Dispositivos interespinhosos}

Esses dispositivos têm o propósito de promover, por meio de técnicas minimamente invasivas (mini-open), a distração entre os processos espinhosos, no sentido de restaurar a altura dos foramens e a estabilização do segmento afetado. Diversos trabalhos foram publicados no lançamento desses dispositivos, mostrando os resultados promissores do seu uso, superiores aos resultados obtidos com a técnica da descompressão simples. ${ }^{44-46}$

Porém, nos últimos anos, revisões sistemáticas e metanálises têm questionado os resultados obtidos, e a North American Spine Society considera que não existem evidências suficientes para indicar a sua utilização, sendo considerada uma técnica ainda investigacional. ${ }^{47-50}$
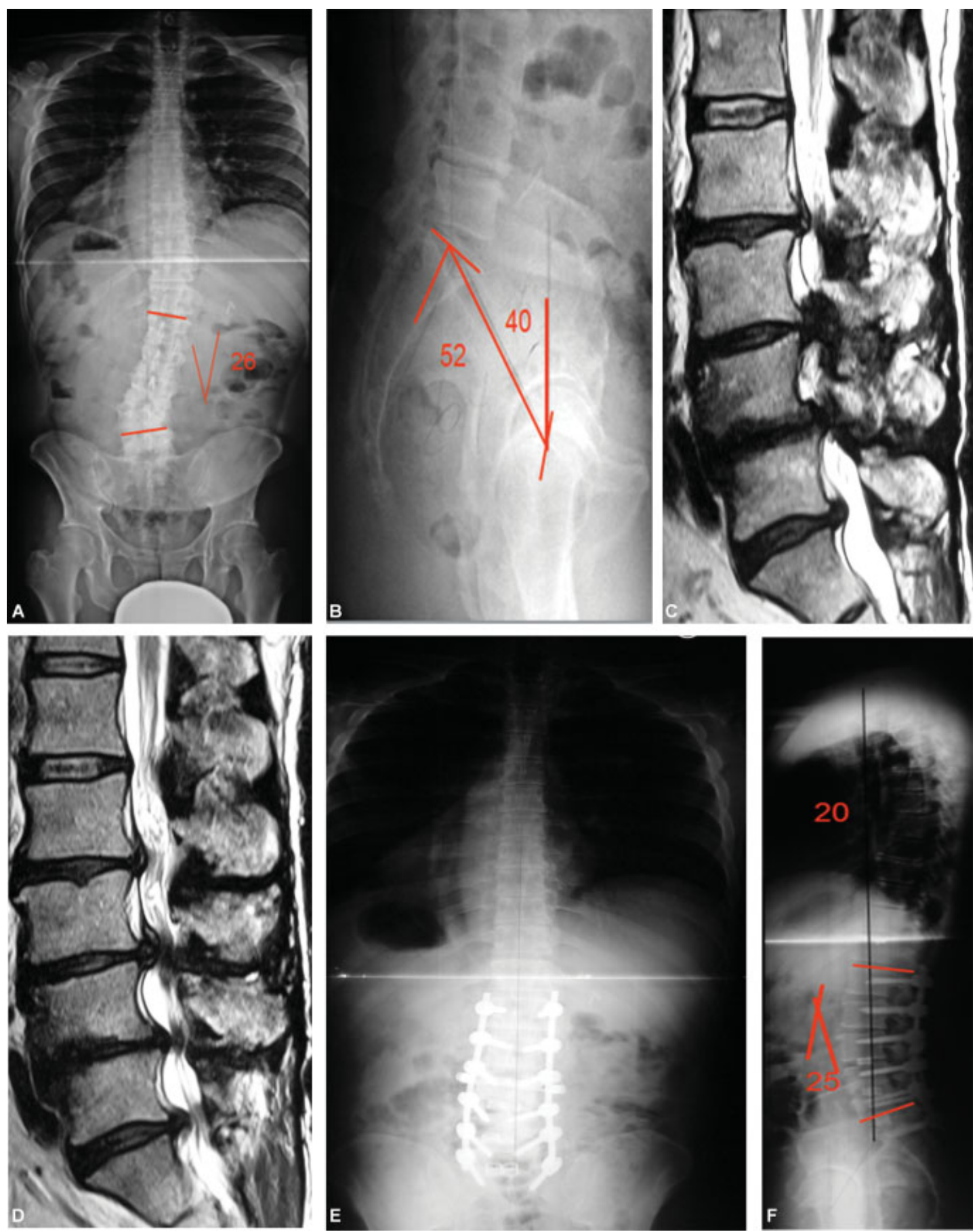

Fig. 3 Escoliose degenerativa: (A e B) Raio-X: escoliose degenerativa com diminuição da lordose lombar e aumento da versão pélvica. (C e D) Corte sagital de ressonância magnética: estenose de canal de L2 a S1. (E e F) Raio-X pós operatório: correção das deformidades com aumento da lordose lombar, diminuição da versão pélvica. 


\section{Escoliose degenerativa}

A escoliose degenerativa é o estágio mais avançado da cascata degenerativa de Kirkaldy-Willis.

A deformidade do eixo coronal não costuma ser acentuada nessas circunstâncias, não ultrapassando, geralmente, os $30^{\circ}$ Cobb. A deformidade do eixo sagital vai se desenvolvendo à medida que há a perda progressiva da lordose lombar, com consequente desequilíbrio do eixo.

É importante o estudo da relação espino-pélvica para avaliar o grau de desequilíbrio do eixo sagital e o grau de versão pélvica que eventualmente já tenha aumentado no sentido de compensar o desequilíbrio. Também é importante o estudo da discrepância da lordose lombar com o grau de incidência pélvica para o planejamento da cirurgia que eventualmente se fará necessária em caso de piora dos sintomas de dor, fadiga muscular, perda de força e dificuldade progressiva de deambulação. Restaurar a lordose lombar é a principal meta na correção da escoliose degenerativa, associada à descompressão dos elementos neurais e a artrodese (-Figura 3A-F). ${ }^{51-55}$

Hoje em dia, a fim de diminuir os riscos de complicações, recomenda-se a associação da artrodese minimamente invasiva por via anterior (anterior lumbar interbody fusion [ALIF, na sigla em inglês]), lateral (extreme lateral interbody fusion [X-LIF, na sigla em inglês]) ou oblíqua (oblique lateral interbody fusion [OLIF, na sigla em inglês] $)^{56-59}$ com a fixação posterior, quando se faz necessário obter grande aumento da lordose lombar, em vez de osteotomia pedicular de subtração posterior.

\section{Considerações finais}

É imperativo conhecer a fisiopatogenia da estenose de canal e determinar a localização precisa da causa da dor pela clínica e pelo estudo por imagem para a indicação do tratamento conservador ou cirúrgico. No que diz respeito à necessidade de tratamento cirúrgico, saber quando indicar somente a descompressão ou associar a artrodese é a meta para obter o melhor resultado.

Conflito de Interesses

Os autores declaram não haver conflito de interesses.

\section{Referências}

1 Eisenstein S. The morphometry and pathological anatomy of the lumbar spine in South African negroes and caucasoids with specific reference to spinal stenosis. J Bone Joint Surg Br 1977; 59(02):173-180

2 Paine KW. Clinical features of lumbar spinal stenosis. Clin Orthop Relat Res 1976;(115):77-82

3 Grobler LJ, Robertson PA, Novotny JE, Ahern JW. Decompression for degenerative spondylolisthesis and spinal stenosis at L4-5. The effects on facet joint morphology. Spine (Phila Pa 1976) 1993;18 (11):1475-1482

4 Turner JA, Ersek M, Herron L, Deyo R. Surgery for lumbar spinal stenosis. Attempted meta-analysis of the literature. Spine (Phila Pa 1976) 1992;17(01):1-8
5 Schönström N, Lindahl S, Willén J, Hansson T. Dynamic changes in the dimensions of the lumbar spinal canal: an experimental study in vitro. J Orthop Res 1989;7(01):115-121

6 Amundsen T, Weber H, Lilleås F, Nordal HJ, Abdelnoor M, Magnaes B. Lumbar spinal stenosis. Clinical and radiologic features. Spine (Phila Pa 1976) 1995;20(10):1178-1186

7 Kirkaldy-Willis WH, Wedge JH, Yong-Hing K, Reilly J. Pathology and pathogenesis of lumbar spondylosis and stenosis. Spine (Phila Pa 1976) 1978;3(04):319-328

8 Liu X, Zhao X, Long Y, Huang K, Xie D, Wang F, et al. Facet Sagittal Orientation: Possible Role in the Pathology of Degenerative Lumbar Spinal Stenosis. Spine (Phila Pa 1976) 2018;43(14):955-958

9 Tomkins-Lane C, Melloh M, Lurie J, et al. ISSLS Prize Winner: Consensus on the Clinical Diagnosis of Lumbar Spinal Stenosis: Results of an International Delphi Study. Spine (Phila Pa 1976) 2016;41(15):1239-1246

10 Delamarter RB, Bohlman HH, Dodge LD, Biro C. Experimental lumbar spinal stenosis. Analysis of the cortical evoked potentials, microvasculature, and histopathology. J Bone Joint Surg Am 1990; 72(01):110-120

11 Rydevik B, Brown MD, Lundborg G. Pathoanatomy and pathophysiology of nerve root compression. Spine (Phila Pa 1976) 1984;9(01):7-15

12 Genevay S, Atlas SJ, Katz JN. Variation in eligibility criteria from studies of radiculopathy due to a herniated disc and of neurogenic claudication due to lumbar spinal stenosis: a structured literature review. Spine (Phila Pa 1976) 2010;35(07):803-811

13 Mamisch N, Brumann M, Hodler J, Held U, Brunner F, Steurer J; Lumbar Spinal Stenosis Outcome Study Working Group Zurich. Radiologic criteria for the diagnosis of spinal stenosis: results of a Delphi survey. Radiology 2012;264(01):174-179

14 Gopinathan P. Lumbar spinal canal stenosis-special features. J Orthop 2015;12(03):123-125

15 Castillo M, Keith Smith J, Mukherji Suresh K. The spine. In: Lee JKT, Segal SS, Stanley RJ, Heiken JP, editors. Computed body tomography with MRI correlation. 3rd. Philadelphia: LippincottRaven; 1998:1451-1454

16 Verbiest H. Neurogenic Intermittent Claudication with Special Reference to Stenosis of the Lumbar Vertebral Canal in Hand Book of Clinical Radiology. Amsterdam: North Holand pub co.; 1976;611:e807

17 Onel D, Sari H, Dönmez C. Lumbar spinal stenosis: clinical/ radiologic therapeutic evaluation in 145 patients. Conservative treatment or surgical intervention? Spine (Phila Pa 1976) 1993; 18(02):291-298

18 Tomkins CC, Dimoff KH, Forman HS, et al. Physical therapy treatment options for lumbar spinal stenosis. J Back Musculoskeletal Rehabil 2010;23(01):31-37

19 Donelson R, Silva G, Murphy K. Centralization phenomenon. Its usefulness in evaluating and treating referred pain. Spine (Phila Pa 1976) 1990;15(03):211-213

20 Moore RA, Straube S, Wiffen PJ, Derry S, McQuay HJ. Pregabalin for acute and chronic pain in adults. Cochrane Database Syst Rev 2009;(03):CD007076

21 Riew KD, Yin Y, Gilula L, et al. The effect of nerve-root injections on the need for operative treatment of lumbar radicular pain. A prospective, randomized, controlled, double-blind study. J Bone Joint Surg Am 2000;82(11):1589-1593

22 Johnsson KE, Udén A, Rosén I. The effect of decompression on the natural course of spinal stenosis. A comparison of surgically treated and untreated patients. Spine (Phila Pa 1976) 1991;16 (06):615-619

23 Zaina F, Tomkins-Lane C, Carragee E, Negrini S. Surgical Versus Nonsurgical Treatment for Lumbar Spinal Stenosis. Spine (Phila Pa 1976) 2016;41(14):E857-E868

24 Kurd MF, Lurie JD, Zhao W, et al. Predictors of treatment choice in lumbar spinal stenosis: a spine patient outcomes research trial study. Spine (Phila Pa 1976) 2012;37(19):1702-1707 
25 Atlas SJ, Keller RB, Wu YA, Deyo RA, Singer DE. Long-term outcomes of surgical and nonsurgical management of lumbar spinal stenosis: 8 to 10 year results from the maine lumbar spine study. Spine (Phila Pa 1976) 2005;30(08):936-943

26 Malmivaara A, Slätis P, Heliövaara M, et al; Finnish Lumbar Spinal Research Group. Surgical or nonoperative treatment for lumbar spinal stenosis? A randomized controlled trial. Spine (Phila Pa 1976) 2007;32(01):1-8

27 Amundsen T, Weber H, Nordal HJ, Magnaes B, Abdelnoor M, Lilleâs F. Lumbar spinal stenosis: conservative or surgical management?: A prospective 10-year study. Spine (Phila Pa 1976) 2000;25(11): 1424-1435

28 Srinivas S, Paquet J, Bailey C, et al. Effect of spinal decompression on back pain in lumbar spinal stenosis: a Canadian Spine Outcomes Research Network (CSORN) study. Spine J 2019;19(06): 1001-1008

29 Rajasekaran S, Thomas A, Kanna RM, Prasad Shetty A. Lumbar spinous process splitting decompression provides equivalent outcomes to conventional midline decompression in degenerative lumbar canal stenosis: a prospective, randomized controlled study of 51 patients. Spine (Phila Pa 1976) 2013;38(20): 1737-1743

30 Cho DY, Lin HL, Lee WY, Lee HC. Split-spinous process laminotomy and discectomy for degenerative lumbar spinal stenosis: a preliminary report. J Neurosurg Spine 2007;6(03):229-239

31 Lee CH, Choi M, Ryu DS, et al. Efficacy and Safety of Fullendoscopic Decompression via Interlaminar Approach for Central or Lateral Recess Spinal Stenosis of the Lumbar Spine: A Metaanalysis. Spine (Phila Pa 1976) 2018;43(24):1756-1764

32 Siepe CJ, Sauer D, Michael Mayer H. Full endoscopic, bilateral overthe-top decompression for lumbar spinal stenosis. Eur Spine J 2018;27(Suppl 4):563-565

33 Rosen DS, O'Toole JE, Eichholz KM, et al. Minimally invasive lumbar spinal decompression in the elderly: outcomes of 50 patients aged 75 years and older. Neurosurgery 2007;60(03):503-509

34 Woo YH, Jung HT, Kim IB, Sun WS, Jung DW. Percutaneous Transforaminal Endoscopic Decompression for Lumbar Foraminal Stenosis. J Clin Exp Orthop3:42

35 Leone A, Guglielmi G, Cassar-Pullicino VN, Bonomo L. Lumbar intervertebral instability: a review. Radiology 2007;245(01): $62-77$

36 da Costa LM, Hennemann AS, de Abreu MR, Antoneli PH. Correlação entre instabilidade radiográfica e presença do sinal de modic. Coluna/Columna 2011;10(02):132-135

37 Hansraj KK, O'Leary PF, Cammisa FP Jr, et al. Decompression, fusion, and instrumentation surgery for complex lumbar spinal stenosis. Clin Orthop Relat Res 2001;200(384):18-25

38 Schroeder GD, Kepler CK, Kurd MF, et al. Rationale for the Surgical Treatment of Lumbar Degenerative Spondylolisthesis. Spine (Phila Pa 1976) 2015;40(21):E1161-E1166

39 Mardjetko SM, Connolly PJ, Shott S. Degenerative lumbar spondylolisthesis. A meta-analysis of literature 1970-1993. Spine (Phila Pa 1976) 1994;19(20, Suppl)2256S-2265S

40 McAnany SJ, Baird EO, Qureshi SA, Hecht AC, Heller JG, Anderson PA. Posterolateral Fusion Versus Interbody Fusion for Degenerative Spondylolisthesis: A Systematic Review and Meta-Analysis. Spine (Phila Pa 1976) 2016;41(23):E1408-E1414

41 Hammad A, Wirries A, Ardeshiri A, Nikiforov O, Geiger F. Open versus minimally invasive TLIF: literature review and metaanalysis. J Orthop Surg Res 2019;14(01):229

42 Pakzaban P. Modified Mini-open Transforaminal Lumbar Interbody Fusion: Description of Surgical Technique and Assessment of Free-hand Pedicle Screw Insertion. Spine (Phila Pa 1976) 2016; 41(18):E1124-E1130
43 Ge DH, Stekas ND, Varlotta CG, et al. Comparative Analysis of Two Transforaminal Lumbar Interbody Fusion Techniques: Open TLIF Versus Wiltse MIS TLIF. Spine (Phila Pa 1976) 2019;44(09): E555-E560

44 Zucherman JF, Hsu KY, Hartjen CA, et al. A multicenter, prospective, randomized trial evaluating the X STOP interspinous process decompression system for the treatment of neurogenic intermittent claudication: two-year follow-up results. Spine (Phila Pa 1976) 2005;30(12):1351-1358

45 Kuchta J, Sobottke R, Eysel P, Simons P. Two-year results of interspinous spacer (X-Stop) implantation in 175 patients with neurologic intermittent claudication due to lumbar spinal stenosis. Eur Spine J 2009;18(06):823-829

46 Richter A, Schütz C, Hauck M, Halm H. Does an interspinous device (Coflex) improve the outcome of decompressive surgery in lumbar spinal stenosis? One-year follow up of a prospective case control study of 60 patients. Eur Spine J 2010;19(02): 283-289

47 Strömqvist BH, Berg S, Gerdhem P, et al. X-stop versus decompressive surgery for lumbar neurogenic intermittent claudication: randomized controlled trial with 2-year follow-up. Spine (Phila Pa 1976) 2013;38(17):1436-1442

48 Poetscher AW, Gentil AF, Ferretti M, Lenza M. Interspinous process devices for treatment of degenerative lumbar spine stenosis: A systematic review and meta-analysis. PLoS One 2018;13(07):e0199623

49 Gazzeri R, Galarza M, Alfieri A. Controversies about interspinous process devices in the treatment of degenerative lumbar spine diseases: past, present, and future. BioMed Res Int 2014; 2014:975052

50 Gazzeri R, Galarza M, Neroni M, et al. Failure rates and complications of interspinous process decompression devices: a European multicenter study. Neurosurg Focus 2015;39(04):E14

51 Yong-Hing K, Kirkaldy-Willis WH. The pathophysiology of degenerative disease of the lumbar spine. Orthop Clin North Am 1983; 14(03):491-504

52 Roussouly P, Gollogly S, Berthonnaud E, Dimnet J. Classification of the normal variation in the sagittal alignment of the human lumbar spine and pelvis in the standing position. Spine (Phila Pa 1976) 2005;30(03):346-353

53 Glassman SD, Berven S, Bridwell K, Horton W, Dimar JR. Correlation of radiographic parameters and clinical symptoms in adult scoliosis. Spine (Phila Pa 1976) 2005;30(06):682-688

$54 \mathrm{Kim}$ YJ, Bridwell KH, Lenke LG, Rhim S, Cheh G. An analysis of sagittal spinal alignment following long adult lumbar instrumentation and fusion to L5 or S1: can we predict ideal lumbar lordosis? Spine (Phila Pa 1976) 2006;31(20):2343-2352

55 Rose PS, Bridwell KH, Lenke LG, et al. Role of pelvic incidence, thoracic kyphosis, and patient factors on sagittal plane correction following pedicle subtraction osteotomy. Spine (Phila Pa 1976) 2009;34(08):785-791

56 Mundis GM, Akbarnia BA, Phillips FM. Adult deformity correction through minimally invasive lateral approach techniques. Spine (Phila Pa 1976) 2010;35(26, Suppl)S312-S321

57 Phillips FM, Isaacs RE, Rodgers WB, et al. Adult degenerative scoliosis treated with XLIF: clinical and radiographical results of a prospective multicenter study with 24-month follow-up. Spine (Phila Pa 1976) 2013;38(21):1853-1861

58 Amaral R, Marchi L, Oliveira L, et al. Opção minimamente invasiva lateral para artrodese intersomática tóraco-lombar. Coluna/ Columna 2011;10(03):239-243

59 Abbasi H, Miller L, Abbasi A, Orandi V, Khaghany K. Minimally invasive scoliosis surgery with oblique lateral lumbar interbody fusion: single surgeon feasibility. Cureus 2017;9(06):e1389 